\title{
Research and Development of Multifunctional Portable Temperature Control Box Based on Semiconductor Refrigeration Technology
}

\author{
Jun $\mathrm{Yu}^{1,}$, , Lihui Sun ${ }^{2, b}$ \\ ${ }^{1}$ Dean`s Office, Jilin Institute of Chemical Technology, Jilin 132022, China \\ ${ }^{2}$ School of Information and Control Engineering, Jilin Institute of Chemical Technology, Jilin \\ 132022, China
}

a83770573@qq.com, bsunlihui2000@126.com

\begin{abstract}
Most of audio players on the market use the hardware filter oAbstract. A multifunctional portable temperature control box based on semiconductor refrigeration technology is designed in order to meet the low and constant temperature requirements of space, military, medical, biological products and so on. This system takes the single chip microcomputer as the control core, uses the semiconductor refrigeration technology, designed the simple, the reliable hardware driving circuit according to the semiconductor TEC device refrigeration principle and the working method unifying the high power MOS tube and the double group of relays, realizes working mode switching and power regulation utilizing the PID and the lag system compensation software control algorithm to control the polarity and switch control of semiconductor TEC devices. The system realizes the $-5^{\circ} \mathrm{C}$ $\sim 60^{\circ} \mathrm{C}$ constant temperature control, and the control precision is high, and it has the characteristics of small size, low cost and clean environment.
\end{abstract}

Keywords: temperature control, semiconductor, TEC.

\section{Introduction}

In recent years, energy shortages and environmental pollution have intensified, countries have begun to prohibit the use of CFCs (Chlorofluorocarbon), which brings a major problem to the refrigeration industry, research and development of environmentally friendly refrigeration technology has become a great challenge for all countries, thus the semiconductor refrigeration technology emerged under this background. Although various types of refrigeration equipment have appeared in the market, but because of its large volume, it is difficult to control the refrigeration temperature accurately. A multifunctional portable temperature control box based on semiconductor refrigeration technology is designed in order to meet the low and constant temperature requirements of space, military, medical, biological products and so on, which realizes the $-5^{\circ} \mathrm{C} \sim 60^{\circ} \mathrm{C}$ constant temperature control and has the high control precision, meanwhile it has clock function and automatic mode conversion function so as to ensure the safe and reliable operation of the system.

\section{Analysis of Semiconductor Refrigeration Principle}

\subsection{Semiconductor Refrigeration Principle}

Semiconductor refrigeration is a successful application of the Peltier effect on semiconductor materials, semiconductors have $\mathrm{N}$ type and $\mathrm{P}$ type, where $\mathrm{N}$ type semiconductors contain excess electrons, thus containing negative temperature difference potentials, while the $\mathrm{P}$ type semiconductor is deficient in electrons, which has excess cavities, and therefore contains positive temperature difference potentials, and connect an $\mathrm{N}$ type semiconductor element and $\mathrm{P}$ type semiconductor element to become a thermocouple forming a P-N knot, when there is direct current going through this $\mathrm{P}-\mathrm{N}$ knot, due to the existence of temperature difference potential, it will produce energy transfer, 
that is, at the junction, will have energy exchange with external environment, when the current reverse, the effect is reversible. The principle of semiconductor refrigeration is shown in Figure 1.

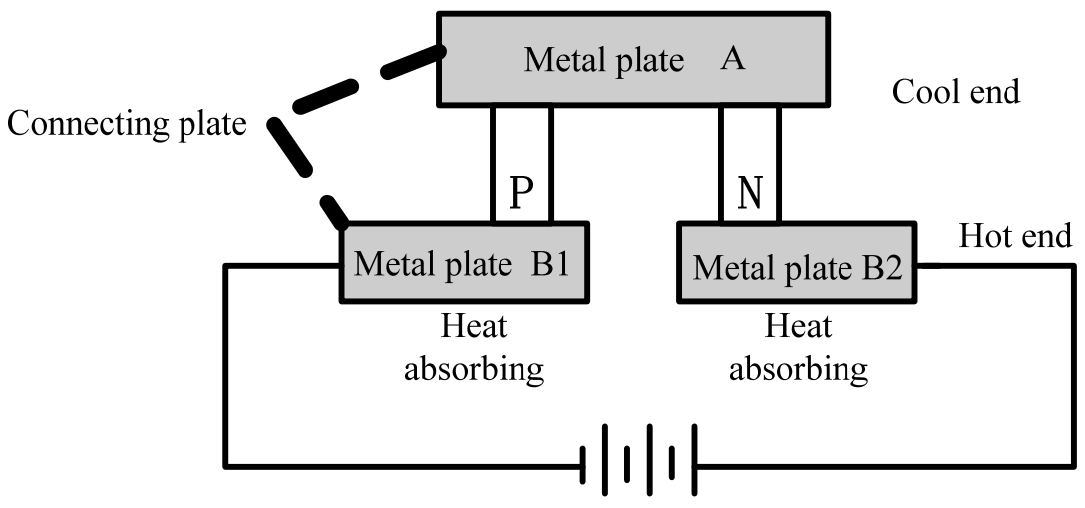

Figure 1. principle diagram of semiconductor refrigeration

\subsection{Semiconductor Refrigeration Simulation}

In order to verify the storage temperature change situation of the temperature control box when the semiconductor refrigerator is in good insulation condition, the heat transfer module in the COMSOL Multiphysics Software Library is used to simulate. First of all, using comsol Drawing command to draw the three-dimensional model of the storage room, and select two of the same model TEC refrigeration plate and place them symmetrically, the box area is one of the refrigeration plate installation location. The application mode of the system is defined as a heat transfer membrane mode in the structure of thin layers and shells, which stipulates that the system property is a heat transfer time variant system. The installation surface of the semiconductor sheet is set to $0^{\circ} \mathrm{C}$ at constant temperature, and the boundary conditions for the rest of the surface are set to adiabatic, assuming the initial ambient temperature is $25^{\circ} \mathrm{C}$. The finite element mesh is automatically generated, and the $3 \mathrm{D}$ model of storage room is shown in Figure 2.

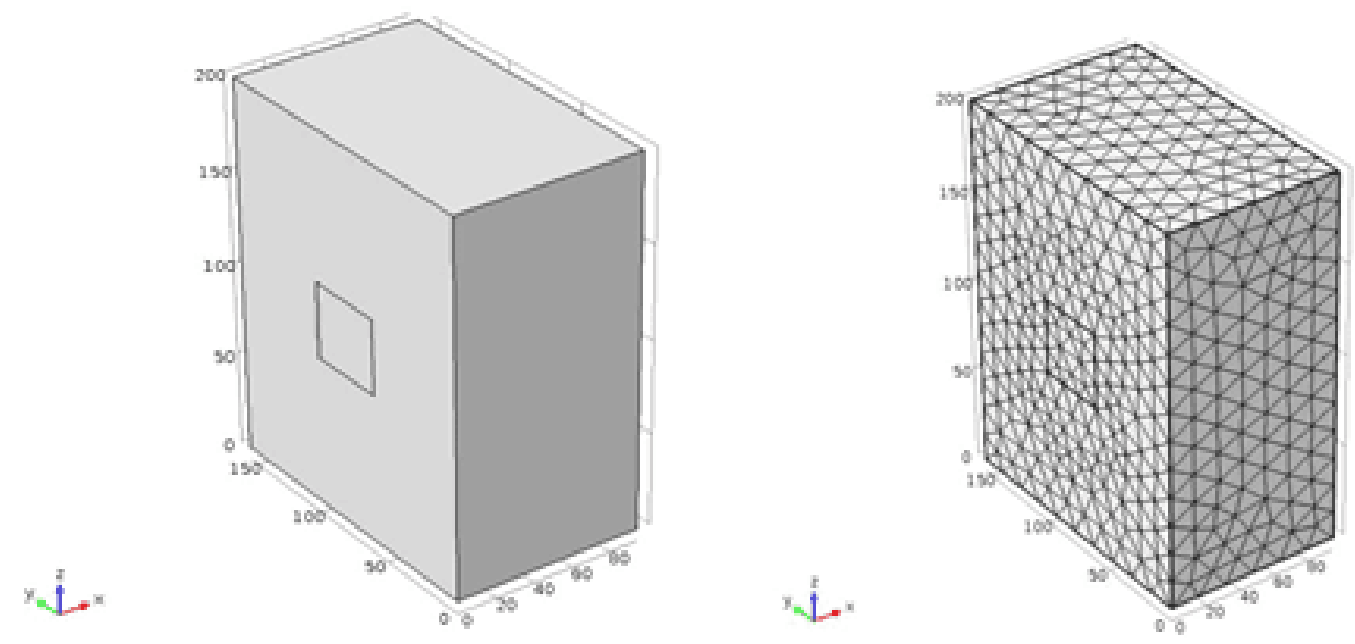

Figure 2. three-dimensional modeling diagram of storage room

In order to control the temperature change evenly, the system design adds the disturbance fan, through increases of the air flow to speed up the temperature control box energy transmission, which is advantageous to the even constant temperature control. The simulation of the cooling effect of the storage room of the temperature control box is shown in Figure 3, in which figures (a), (b) and (c) represent the control temperature change section of temperature control box storage room when $\mathrm{t}=500 \mathrm{~s}, \mathrm{t}=1000 \mathrm{~s}$ and $\mathrm{t}=1500 \mathrm{~s}$ respectively. According to the simulation results, the design of temperature control box based on semiconductor refrigeration technology can realize the requirement of design function, and the theoretical analysis and practical scheme are feasible. 


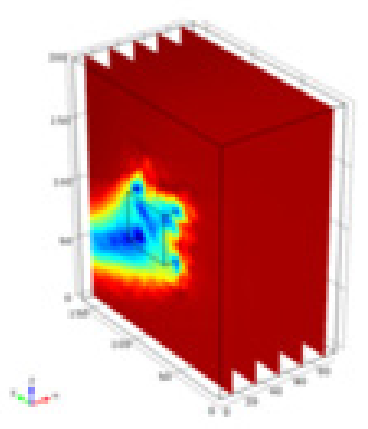

(a) $\mathrm{t}=500 \mathrm{~s}$
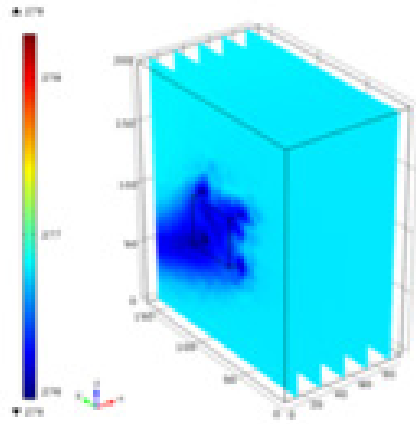

(b)t $=1000 \mathrm{~s}$
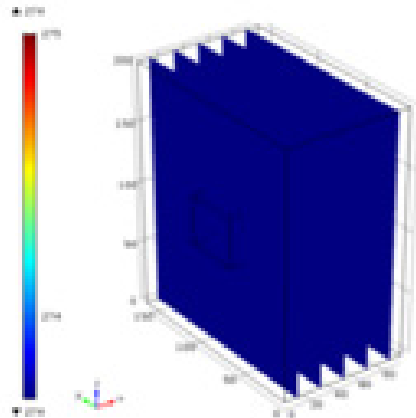

(c) $\mathrm{t}=1500 \mathrm{~s}$

Figure 3. Control temperature Simulation change diagram

\section{The Overall Design Scheme of the System}

The multi-function portable temperature control box mainly realizes low temperature constant temperature control under special environment. The overall structure design of the system includes controller, TEC semiconductor refrigerator, temperature sensor, input and output interface, indicating module and so on. In practical application, a single chip microcomputer is used as the controller, the temperature is detected in real time according to the temperature sensor, and the polarity and power of TEC semiconductor is controlled by the driving circuit to realize constant temperature control. At the same time, the system uses key to input parameters settings and outputs detection and control information with the LCD liquid crystal display device. The overall structure of the system is shown in Figure 4.

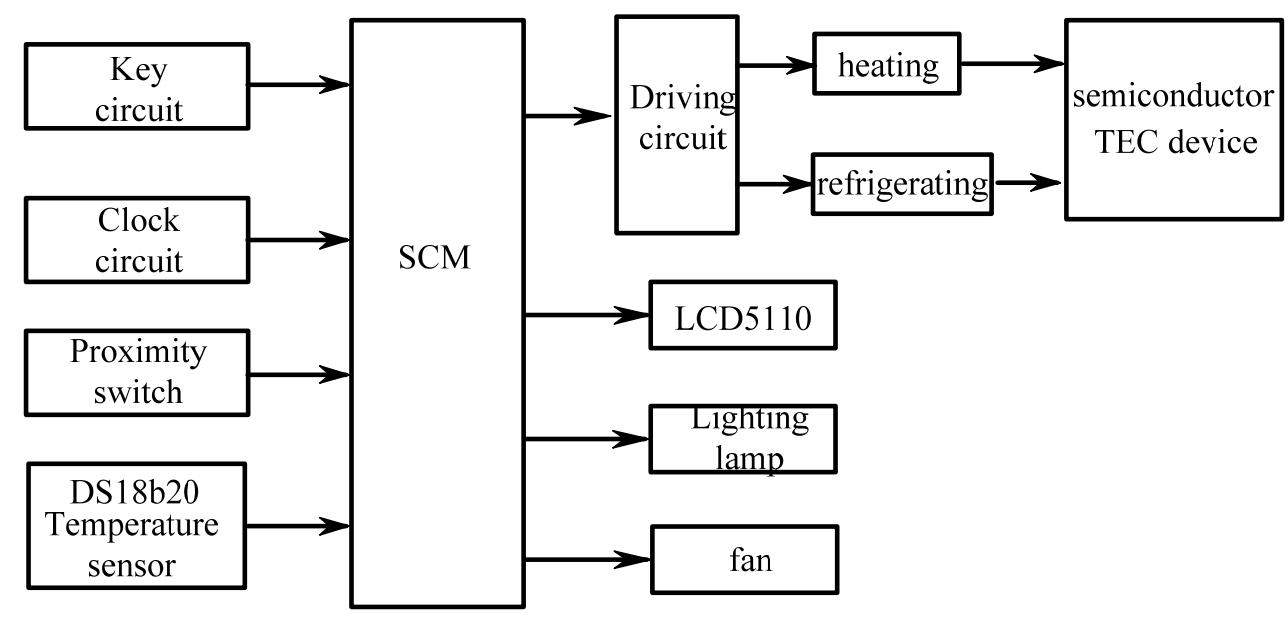

Figure 4. overall structure diagram of the system

\section{Hardware Design of the System}

The design of the system hardware circuit mainly realizes the drive control of each device and equipment, including the power supply circuit, the temperature detection circuit, the TEC drive circuit, the control signal circuit, the liquid crystal circuit, the key circuit, the clock circuit, the lighting and the fan control circuit. The principle of circuit design is to meet the working principle of each component, to meet the standard of working voltage or current. At the same time, it needs to meet the requirements of the overall coordinated control of the system, and has working characteristics of safety, reliability and stability. 


\subsection{Power Supply Circuit}

The work of temperature control box control system needs two kinds of voltage power supply of $+12 \mathrm{~V}$ and $+5 \mathrm{~V}$, of which $12 \mathrm{~V}$ power supply is mainly for semiconductor TEC refrigerator, heat radiation water pump, disturbance fan and lighting lamp. $5 \mathrm{~V}$ power supply mainly applies for SCM controller, temperature sensor, liquid crystal display screen, clock circuit and so on. Because semiconductor TEC devices are high power devices with high working current, in order to increase the system reliability, $12 \mathrm{~V}$ switching power supply should be selected. The application of $5 \mathrm{~V}$ voltage power supply is obtained by reducing $12 \mathrm{~V}$ power, through the use of capacitive filter and the threeend integrated voltage stable voltage regulator LM7805 to stabilize, then output stable $+5 \mathrm{~V}$ DC power supply to supply power for the MCU controller and other devices.

\subsection{Temperature Detection Circuit}

The temperature detection circuit is the application circuit of the temperature sensor and realizes the real-time and high-precision detection of the temperature signal. Because DS18b20 is a single bus digital temperature sensor, it does not need signal conditioning circuit and so on. At the same time, the single bus device has a high requirement for the electric level of control signal. In order to ensure the stability and reliability of the transmission signal of the temperature sensor, it needs to connect the pull resistance between the control signal pin and the VCC, and the practical application circuit is as shown in Figure 5.

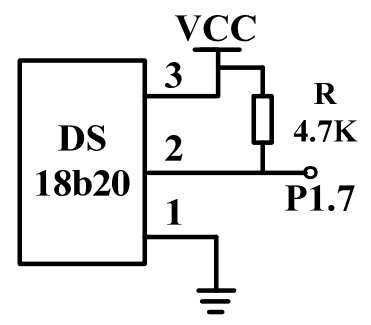

Figure 5. temperature detection circuit

\subsection{The Design of the Driving Circuit of the TEC Refrigerator}

According to the working principle of the semiconductor refrigerator TEC, it is necessary to control the polarity and power of the power supply flexibly. At the same time, because of the high current characteristic of the TEC, the design of the driving circuit needs to consider the power loss and a large number of heat problems generated [5-8]. By analyzing the working principle of TEC semiconductor devices and the requirements of control function, a simple and reliable TEC device driver circuit is designed in combination with the functions of double groups of relays and high-power MOS tubes. The actual drive circuit is shown in Figure 6.

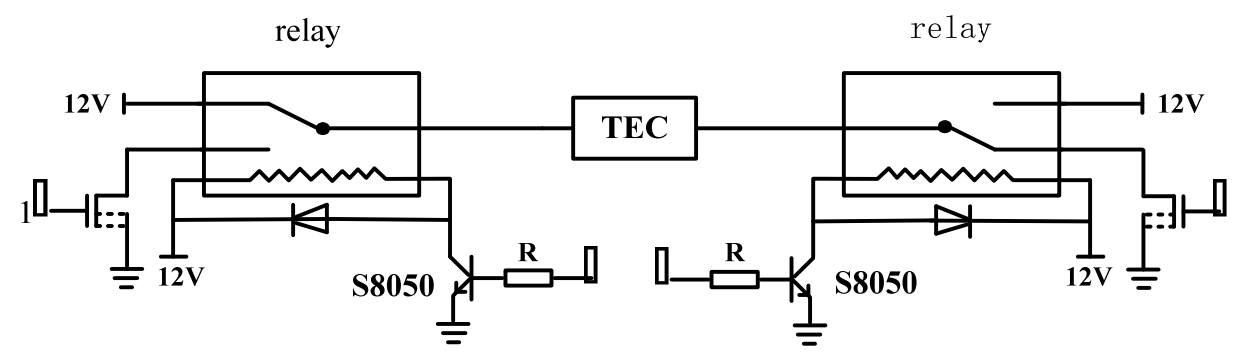

Figure 6. TEC refrigerator driving circuit

\subsection{Control Signal Circuit}

The control signal circuit is to realize the output control signal of SCM to control equipment, mainly to the mode control of double groups of relays and the switch control of MOS tube, that is to 
control the working mode and working power of Semiconductor TEC refrigerator. The design circuit includes isolation of optocoupler signals to prevent interference of high-power working devices to SCM controllers, and a circuit for signal electric level conversion. The design of the control signal circuit is shown in Figure 7.

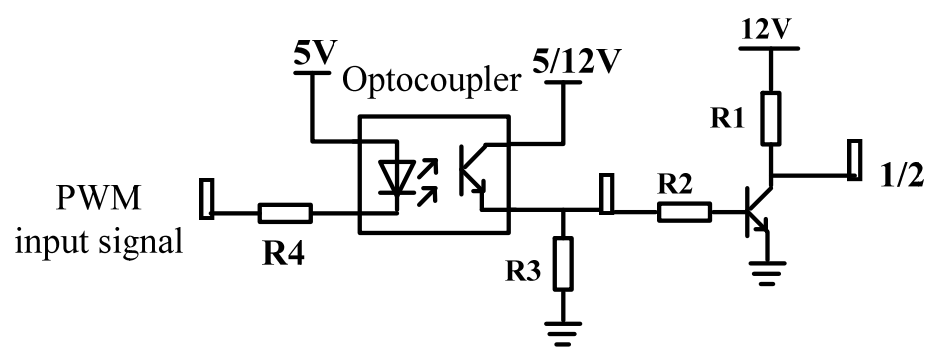

Figure 7. control signal circuit

\subsection{Control Circuit of Lighting Lamp and Disturbance Fan}

Lighting lamp and disturbance fan are important parts of the function design of the temperature control box, when opening the box door, it need to turn on the light, the function of the disturbance fan is to disturb the air in the control room when close the box door, making the control temperature even. The working state of two devices is opposite to each other, and the control selection is based on the proximity switch input signal. The system design uses the relay to switch the control switch, uses the output IO signal to control the triode to drive the relay, so as to complete the corresponding function setting. The design of the control circuit is as shown in Figure 8.

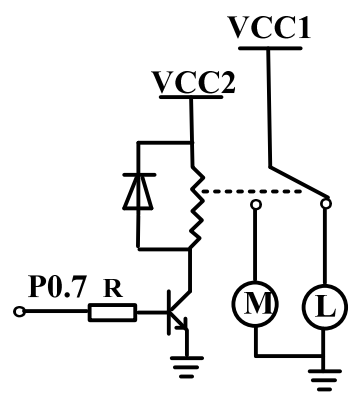

Figure 8. light and fan control circuit

\section{Software Design of the System}

The reasonable control flow is the requirement of the function design of the temperature box, and it is also the key to ensure the stable and reliable work of the system. The design of the system mainly controls the working mode and power of TEC semiconductor devices to achieve the goal of setting the temperature environment. The design of the temperature control system is based on the practical application and makes the system work and operation intelligent to the maximum convenience of practical application, on the basis of guaranteeing the basic temperature controlling function requirement.

According to the specific equipment flow control, the control algorithm is used to control the coordination of each link, and the control flow of the specific equipment is shown in Figure 9. 


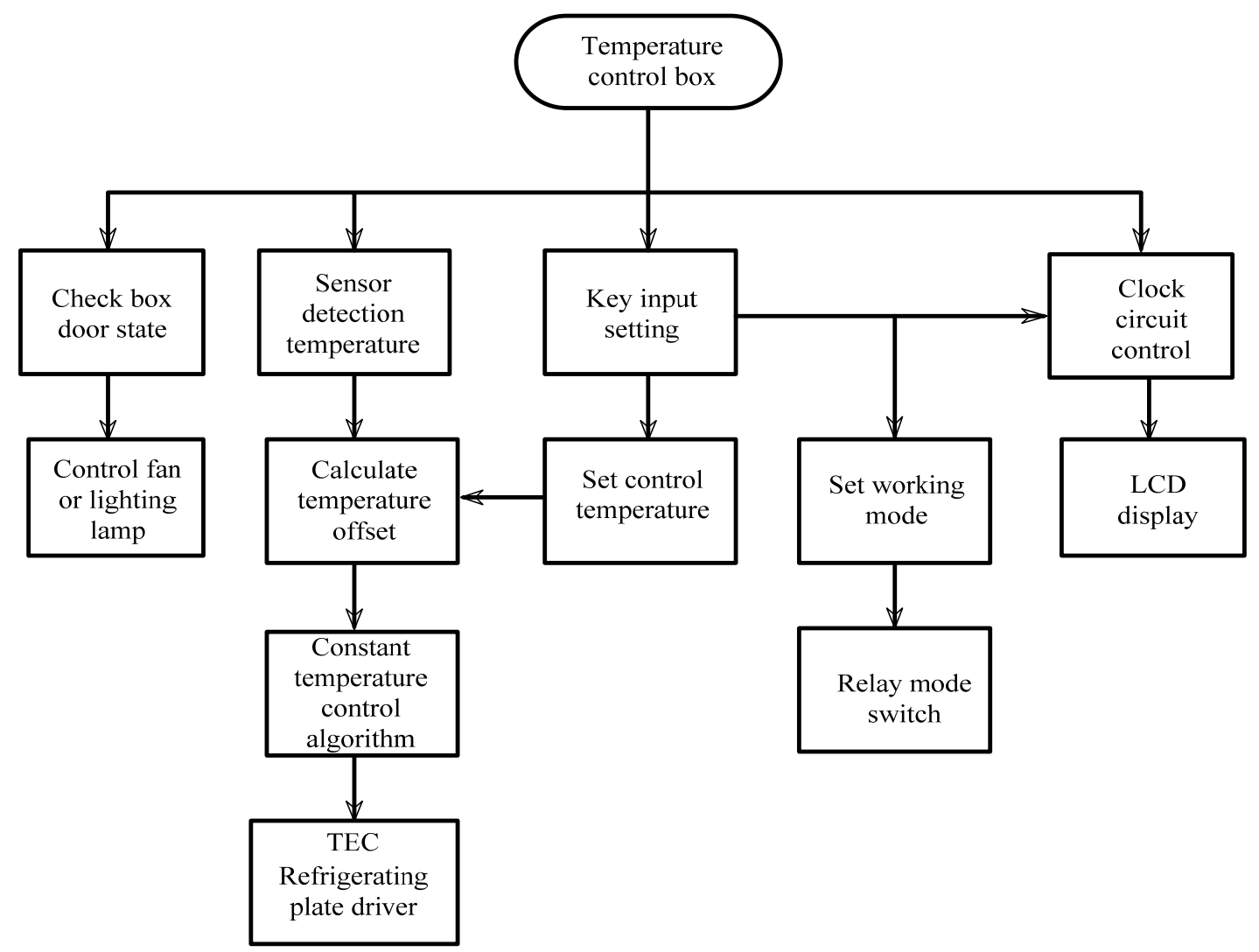

Figure 9. Control flow Chart of temperature control box

\section{Function Tests of the System}

After the program demonstration of the control system and the actual software and hardware design, the function of the product is tested. First, the functions of each component of the system are tested, including the function test of the temperature sensor, the output of liquid crystal display, the function of the input operation, the control effect of the TEC device and so on. At the same time, the voltage value of the key control points of the circuit board is measured, and the operation state of system is analyzed by the obtained the data information to ensure that the temperature control box works faultlessly, safely and reliably. Figure 10 shows the display information of the basic operation state of the temperature control box.

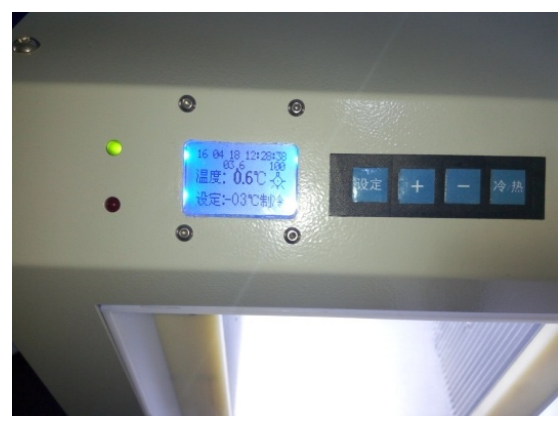

Figure 10. information diagram of the basic operating state of the temperature control box

According to the diagram result, the detected temperature of temperature sensor is $0.6^{\circ} \mathrm{C}$, the refrigeration working mode was set, the constant temperature control target is $-3^{\circ} \mathrm{C}$, the equipment operation state is normal, the clock circuit is working normally, this indicates that the temperature control box realizes the function design request of each part. After a long period of control, the actual control effect of the comparison as shown in Figure 11, it is clearly shown the work effect of the system in the refrigeration mode. 


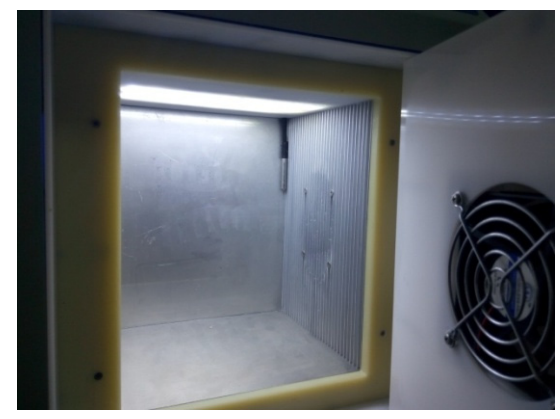

Figure 11. refrigeration effect diagram

\section{Conclusion}

The design of multifunctional portable temperature control box system based on semiconductor refrigeration technology, from the practical application point of view, facing the important demand in special environment, the constant temperature controlling function of heating /refrigeration mode is realized by using semiconductor refrigeration technology. The design scheme of the whole work is divided into two parts: hardware circuit and software program. After the refrigeration technology verification and the design scheme demonstration, carries on the actual product design and the production, and realizes the goals of function request and the control precision.

\section{Acknowledgments}

This work was financially supported by Key project of Jilin Institute of Chemical Engineering 2017079.

\section{References}

[1]. Tang Yalin, Xu Zhiliang. Key technology analysis of semiconductor refrigeration and air conditioning design $[\mathrm{J}]$. Refrigeration and air conditioning, 2015, 15 (7): 1 - 4.

[2]. Qi Hongfang. Study on semiconductor temperature control system [J]. Journal of Lanzhou Institute of Industry, 2013, 02: 13 - 16.

[3]. Chen Xueqing, Tong Lei, Liu Jixin, et al. Experimental research on portable solar heating and cooling package based on semiconductor technology [J]. Refrigeration, 2016, 137 (35): 70 - 75. 